\title{
Acute Cardiac Bradyarrhythmias during Pituitary Surgery: What Should We Know?
}

\author{
Vishal Devra ${ }^{1}$ Shalvi Mahajan \\ ${ }^{1}$ Department of Anaesthesia and Intensive Care, Postgraduate \\ Institute of Medical Education and Research, Chandigarh, India
}

J Neurosci Rural Pract:2020;11:504-505

Surgery of the pituitary tumor poses unique challenges to neuroanesthesiologists and neurosurgeons owing to associated intraoperative hemodynamic disturbances. We report a case of acute cardiac arrhythmia causing hemodynamic disturbances that occurred due to pituitary surgery and detail the measures taken during its management.

A 28-year-old female, weighing $55 \mathrm{~kg}$, was diagnosed with nonfunctional pituitary macroadenoma with apoplexy and was scheduled for transnasal, transsphenoid endoscopic resection. Her primary complaints were diminished visual acuity, galactorrhea, amenorrhea, headache, and vomiting. She did not have any cardiorespiratory abnormality, and her systemic examination and routine investigations were normal. The standard general anesthesia technique was followed. For induction, morphine $6 \mathrm{mg}$, propofol $2 \mathrm{mg} / \mathrm{kg}$, and vecuronium $1 \mathrm{mg} / \mathrm{kg}$ were used, and maintenance of anesthesia was achieved using oxygen-nitrous oxide-desflurane mixture along with intermittent doses of vecuronium. Intraoperatively, following an initial 1.5 hours of stable hemodynamics, the patient's heart rate suddenly dropped to $\leq 40$ beats per minute, with sinus rhythm and normal QRS morphology in electrocardiogram associated with drop-in invasive blood pressure ( $\bullet$ Fig. 1A). The surgeon was informed immediately; further stimulus was stopped and surgery restarted after normalization of vitals. A similar event reoccurred thrice and everytime stimulus was stopped by the surgeon ( - Fig. 1B, C). Nonetheless, the pharmacological intervention was never required. On interrogation, the surgeon informed that they were operating near the optic chiasma. Hence, it was decided to go ahead for the surgery with a gradual and gentle approach near the chiasmal part of the tumor. At the end of the surgery, the patient was extubated following the reversal of neuromuscular blockade. Furthermore, stay in the hospital was uneventful.Sudden cardiac bradyarrhythmias and acute hemodynamic changes during pituitary surgery can be because of preoperative and intraoperative causes. Preoperative factors could be hypertension or cardiac disease, medications such as $\beta$-blockers, or the use of somatostatin analog such as octreotide. Intraoperatively, anesthesia-related or surgical

\begin{abstract}
Address for correspondence Shalvi Mahajan, MD, DM, Department of Anaesthesia and Intensive Care, Postgraduate Institute of Medical Education and Research, 4th Floor, Nehru Building, Chandigarh 160012, India (e-mail: drshalvimahajan@gmail.com).
\end{abstract}

causes might be responsible. Anesthesia-related causes include lighter plane of anesthesia, inadequate analgesia, hypothermia, an overdose of inhalational or intravenous anesthetic agents, and drug errors, as well as the use of hypotensive anesthesia (dexmedetomidine, a commonly used drug nowadays). Surgical factors pertinent to pituitary surgery are compression or manipulation of carotid vessels, dural stretching, manipulation of the hypothalamic region, stimulation of the trigeminal nerve, acute hydrocephalus, sudden blood loss, and venous air embolism. In our case, there were no signs of inadequate anesthesia/ analgesia, sudden blood loss, hypothermia, and fall in end-tidal carbon dioxide. Acute hydrocephalus can cause hypertension and bradycardia through Cushing's response, but it was unlikely in our case as there was bradycardia with hypotension. Hence, the most probable event responsible for sudden bradycardia and hypotension in our case was because of the activation of a trigeminal cardiac reflex (TCR) through optic chiasmal compression, which further supported by a cause-effect relationship. ${ }^{1}$

TCR is a well-known biological reflex. Coexisting risk factors such as hypoxia, hypercarbia, acidosis, young age, a lighter plane of anesthesia, strong and prolong provoking stimulus, and preoperative medications can render patients more vulnerable to TCR in the perioperative phase.

Stimulation of divisions of the trigeminal nerve during its intracranial course can cause bradycardia and/or asystole due to TCR in transsphenoidal surgeries. ${ }^{2}$ Sudden bradycardia, hypotension, apnea, and gastric hypermobility are manifestations of the TCR. ${ }^{3}$ There are two forms of TCR (i.e., peripheral and central) described that cause bradycardia with acute blood pressure changes. ${ }^{3}$ However, peripheral TCR (triggered through the extracranial part of the trigeminal nerve) can cause hypertension as compared with hypotension in central TCR (triggered through central divisions of the trigeminal nerve including Gasserian ganglion).,34

Life-threatening events following TCR occurs rarely, but during the literature search, we found one similar case published by Chowdhury et al where three episodes of bradycardia occurred during endoscopic transsphenoidal surgery while 


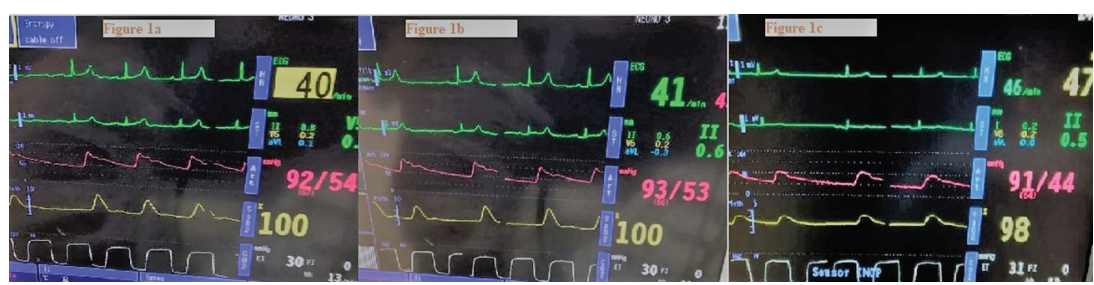

Fig. 1 Electrocardiogram of the patient.

operating near optic chiasma, the patient developed vision loss in the postoperative period, and hematoma was seen on imaging in the sellar region compressing the optic chiasma, which required evacuation. Thus, indicating that intraoperative TCR may act as a warning indicator and may predict surgical outcome albeit in our case postoperative course was uneventful unlike reported by Chowdhury et al.

Through this case report, we want to highlight that TCR is mostly a benign biological reflex, but the neuroanesthesia team should be vigilant of its occurrence. Proper communication and meticulous surgical dissections while operating in or near the optic chiasma will improve the overall success rate of the surgery.

\section{Conflict of Interest}

None declared.

\section{References}

1 Meuwly C, Golanov E, Chowdhury T, Erne P, Schaller B. Trigeminal cardiac reflex: new thinking model about the definition based on a literature review. Medicine (Baltimore) 2015;94(5):e484

2 Cho JM, Min KT, Kim EH, Oh MC, Kim SH. Sudden asystole due to trigeminocardiac reflex during transsphenoidal surgery for pituitary tumor. World Neurosurg 2011;76(5):477. e11-477.e15

3 Chowdhury T, Mendelowith D, Golanov E, et al; TrigeminoCardiac Reflex Examination Group. Trigeminocardiac reflex: the current clinical and physiological knowledge. J Neurosurg Anesthesiol 2015;27(2):136-147

4 Singh GP, Chowdhury. T. Brain and heart connections: the trigeminocardiac reflex! J Neuroanesthesia Critical Care 2017;4(2):71-77 